\title{
Addendum
}

\section{PEG-Supported Synthesis of 3,5-Disubstituted-1,2,4-Triazoles}

Jun-Ke Wang,* Ying-Xiao Zong, Guo-Ren Yue Synlett 2005, 1135.

The following references should be added to this communication, and should be cited immediately following the mention of Figure 1: (8) (a) Samanta, S. K.; Yli-Kauhaluoma, J. J. Comb. Chem. 2005, 7, 142. (b) Bilodeau, M. T.; Cunningham, A. M. J. Org. Chem. 1998, 63, 2800. 